\title{
CircHIPK3 knockdown suppresses E2F3 expression to inhibit the viability and promote the apoptosis of lens epithelial cells in age-related cataract by sponging miR-499a-5p
}

\author{
Type \\ Research paper

\section{Keywords} \\ E2F3, circHIPK3, miR-499a-5p, apoptosis, Age-related cataract
}

\begin{abstract}
Introduction

Circular RNA (circRNA) is considered to be a vital regulator of disease progression, including agerelated cataract (ARC). However, the molecular mechanism of circHIPK3 in ARC progression has not been fully elucidated.

Material and methods

The expression levels of circHIPK3, miR-499a-5p and E2F transcription factor 3 (E2F3) were measured by quantitative real-time PCR. Cell counting kit 8 assay and flow cytometry were performed to detect cell viability and apoptosis, respectively. Western blot analysis was employed to test the protein levels of apoptosis-related markers and E2F3. Biotin-labeled RNA pull-down assay was used to select miRNAs that could be targeted by circHIPK3, and dual-luciferase reporter assay was performed to confirm the interaction between miR-499a-5p and circHIPK3 or E2F3.
\end{abstract}

\section{Results}

CircHIPK3 is a stable circRNA that is significantly under-expressed in the anterior lens capsule tissues of ARC patients. Knockdown of circHIPK3 suppressed SRA01/04 cell viability and accelerated apoptosis. Moreover, miR-499a-5p could be targeted by circHIPK3, and its inhibitor reversed the effect of circHIPK3 silencing on cell viability and apoptosis. Furthermore, E2F3 was a target of miR-499a-5p, and its overexpression reversed the effect of miR-499a-5p on the viability and apoptosis of SRA01/04 cells. In addition, circHIPK3 positively regulated E2F3 expression by sponging miR-499a-5p.

\section{Conclusions}

CircHIPK3 knockdown inhibited viability and enhanced apoptosis of lens epithelial cells to promote ARC progression by regulating miR-499a-5p/E2F3 axis. 
CircHIPK3 knockdown suppresses E2F3 expression to inhibit the viability and promote the apoptosis of lens epithelial cells in age-related cataract by sponging miR-499a-5p

Qichao Han, Rong Zhang, Lan Ma, Li Shao, Meiyan Feng*

Department of Ophtalmology, Zaozhuang Municipal Hospital Shandong Province, Zaozhuang 277100, Shandong, China.

*Corresponding Author: Meiyan Feng, Department of Ophtalmology, Zaozhuang Municipal Hospital Shandong Province, No. 41 Longtou Road, Shizhong District, Zaozhuang 277100, Shandong, China. Tel: +86-0632-3227207. Email: zjq33yb@163.com. 


\begin{abstract}
Introduction: Circular RNA (circRNA) is considered to be a vital regulator of disease progression, including age-related cataract (ARC). However, the molecular mechanism of circHIPK3 in ARC progression has not been fully elucidated.
\end{abstract}

Methods: The expression levels of circHIPK3, miR-499a-5p and E2F transcription factor 3 (E2F3) were measured by quantitative real-time PCR. Cell counting kit 8 assay and flow cytometry were performed to detect cell viability and apoptosis, respectively. Western blot analysis was employed to test the protein levels of apoptosis-related markers and E2F3. Biotin-labeled RNA pull-down assay was used to select miRNAs that could be targeted by circHIPK3, and dual-luciferase reporter assay was performed to confirm the interaction between miR-499a-5p and circHIPK3 or E2F3.

Results: CircHIPK3 is a stable circRNA that is significantly under-expressed in the anterior lens capsule tissues of ARC patients. Knockdown of circHIPK3 suppressed SRA01/04 cell viability and accelerated apoptosis. Moreover, miR-499a-5p could be targeted by circHIPK3, and its inhibitor reversed the effect of circHIPK3 silencing on cell viability and apoptosis. Furthermore, E2F3 was a target of miR-499a-5p, and its overexpression reversed the effect of miR-499a-5p on the viability and apoptosis of SRA01/04 cells. In addition, circHIPK3 positively regulated E2F3 expression by sponging miR-499a-5p.

Conclusion: CircHIPK3 knockdown inhibited viability and enhanced apoptosis of lens epithelial cells to promote ARC progression by regulating miR-499a-5p/E2F3 axis.

Keywords: Age-related cataract, apoptosis, circHIPK3, miR-499a-5p, E2F3 


\section{Introduction}

Age-related cataract (ARC) is the most common type of cataract, which is more common in middle-aged and older people over 50 years of age $[1,2]$. The etiology of ARC is more complicated, which may be due to physiological aging, malnutrition and genetic factors $[3,4]$. In addition, at the molecular level, abnormal apoptosis of lens epithelial cells (LECs) caused by various factors is considered to be the direct cause of ARC [5, 6]. At present, surgical treatment is still the most basic and effective means of ARC treatment [7]. However, the occurrence of postoperative complications has also seriously affected the quality of life of patients [8]. Therefore, there is still necessary to find the potential mechanisms that affect the occurrence of ARC to provide new evidence for slowing the progression of ARC.

Circular RNAs (circRNAs) are non-coding RNA molecules that do not have 5'end caps and 3' end poly(A) tails and form circular structures by covalent bonds in living organisms [9, 10]. With the deepening of research, circRNA has been found to be an important player in cell biology process and disease development $[11,12]$. CircRNA has been shown to be a promising biomarker for ocular diseases [13]. For example, circKMT2E has been reported to be up-regulated in diabetic cataract and may be related to the pathogenesis of DC [14]. CircHIPK 3 has been found to be differentially expressed in many diseases and is associated with disease progression in recent years $[15,16]$. Some studies have confirmed that circHIPK 3 is significantly up-regulated in diabetes mellitus and can promote retinal vascular dysfunction [14]. In ARC, circHIPK3 was demonstrated to be significantly down-regulated and was associated with the proliferation and apoptosis of LECs [17]. Therefore, its role in 
ARC development is worth further exploration.

CircRNA contains a large number of microRNA (miRNA) binding sites and can function as "miRNA sponge", which indirectly regulates the expression of downstream targets of miRNA $[18,19]$. Therefore, the circRNA-miRNA-mRNA axis is an important way to elucidate the molecular mechanism of circRNA. The purpose of this study is to explore the role of circHIPK3 in ARC progression and to explore its potential molecular mechanism through the circRNA-miRNA-mRNA axis, thus providing a more reliable theoretical basis for mitigating ARC progression.

\section{Materials and methods}

\section{Clinical tissues}

ARC patients ( 9 men/11 women, age range $56-72$ years $)$ and donors (12 men/8 women, age range 55-71 years) in this study were recruited from Zaozhuang Municipal Hospital Shandong Province. Inclusion criteria: lens was opaque, visual acuity was $\leqslant 0.6$, and double lacrimal passage was unobstructed and free of other ocular diseases. Exclusion criteria: other ocular diseases, high myopia, ocular trauma, diabetes, ocular inflammation and women during pregnancy or lactation. 20 eyes of normal anterior lens capsule tissues (Control) were collected within 10 hours of the donor's death, while 20 eyes of anterior lens capsule tissues were obtained during routine surgery in ARC patients. Informed consent was obtained from each patient and donor. All procedures were conducted in accordance with the principles of the Helsinki declaration and were approved by the Ethics Committee of Zaozhuang Municipal Hospital Shandong Province.

\section{Cell culture and transfection}


Human LECs (SRA01/04) were obtained from Biovector (Beijing, China) and cultured in Dulbecco's modified Eagle's medium (DMEM; Gibco, Carlsbad, CA, USA) supplemented with $10 \%$ fetal bovine serum (FBS; Gibco) and 1\% penicillin/streptomycin (Invitrogen, Carlsbad, CA, USA) at $37^{\circ} \mathrm{C}$ in an incubator with $5 \% \mathrm{CO}_{2}$.

Small interference RNA against circHIPK3 (si-circHIPK3), miR-499a-5p mimic and inhibitor (miR-499a-5p and anti-miR-499a-5p), pcDNA3.1 circHIPK3 and E2F transcription factor 3 (E2F3) overexpression vector (circHIPK3 and E2F3), and negative controls (si-NC, miR-NC, anti-miR-NC and vector) were obtained from General Biosystems (Anhui, China). The above oligonucleotides $(50 \mathrm{nM})$ or vectors $(4.0 \mu \mathrm{g})$ were transfected into SRA01/04 cells using Lipofectamine 3000 (Invitrogen) when cells reached 70\% confluences (did not undergo serum deprivation). After transfection for $48 \mathrm{~h}$, the cells were harvested for function analysis.

\section{Quantitative real-time PCR (qRT-PCR)}

TRIzol reagent (Invitrogen) was used to isolate total RNA. After reverse-transcribed to cDNA using cDNA Synthesis Kit (Takara, Dalian, China), SYBR Premix Dimer Eraser kit (Takara) was used to perform qRT-PCR in PCR system (Applied Biosystems, Foster, CA, USA). GAPDH and U6 were used as the internal controls. The primers were shown as below: circHIPK3, F 5'-TTCAACATATCTACAATCTCGGT-3', $\quad$ R 5'-ACCATTCACATAGGTCCGT-3'; HIPK3, F 5'-TGGAGACTGGGGGAAGATGA-3', R 5'-CACACTAACTGGCTGAGGGG-3'; E2F3, F 5'-CACTTCCACCACCTCCTGTT-3', R 5'-TGACCGCTTTCTCCTAGCTC-3'; GAPDH, F 5'-GGGAGCCAAAAGGGTCAT-3', R 5'-GAGTCCTTCCACGATACCAA-3'; miR-499a-5p, F 5'-GCCGAGTtAAGACTTGCAGTG-3'， R 5'-CAGTGCGTGTCGTGGAGT-3'; U6， F 
5'-CTCGCTTCGGCAGCACA-3'， R 5'-AACGCTTCACGAATTTGCGT-3'. The $2^{-\Delta \Delta C t}$ method was used to quantify relative expression.

\section{Actinomycin D (ActD) assay}

ActD was obtained from AAT Bioquest (Sunnyvale, CA, USA). In brief, SRA01/04 cells were incubated with ActD for 0, 4, 8, 12 and 24 h, respectively. Then, circHIPK3 and HIPK3 expression levels were detected using qRT-PCR. Linear HIPK3 was used as negative control. For detecting the effect of circHIPK3 on the degradation of miR-499a-5p, SRA01/04 cells were transfected with circHIPK3 overexpression vector or vector and then incubated with ActD for 0,12 and $24 \mathrm{~h}$, respectively. The expression of miR-499a-5p was measured by qRT-PCR.

\section{Ribonuclease R (RNase R) assay}

After extracted RNA using TRIzol reagent, the RNA $(2.0 \mu \mathrm{g})$ was treated with RNase R (Geneseed, Guangzhou, China) for 15 min. The RNA without RNase R treatment was used as negative control (Mock). After that, circHIPK3 and liner HIPK3 expression was detected by qRT-PCR.

\section{Subcellular fractionation assay}

The cytoplasm and nuclear RNA of SRA01/04 cells were extracted using Cytoplasmic \& Nuclear RNA Purification Kit (Norgen Biotek Corp, Ontario, Canada). U6 was used as nuclear control and GAPDH was used as cytoplasm control. QRT-PCR was performed to test the expression levels of circHIPK3, U6 and GAPDH in the cytoplasm and nuclear of SRA01/04 cells.

\section{Cell counting kit 8 (CCK8) assay}


After transfection, SRA01/04 cells were harvested and re-inoculated in 96-well plates $\left(1 \times 10^{3}\right.$ cells/well). After 48 h, CCK8 solution (Dojindo, Tokyo, Japan) was added into each well and incubated for $2 \mathrm{~h}$. The absorbance was detected at $450 \mathrm{~nm}$ using a microplate reader to evaluate cell viability.

\section{Flow cytometry}

SRA01/04 cells were digested with trypsin after transfection, and the cell suspension was collected $\left(1 \times 10^{5}\right.$ cells). Then, Annexin V-fluorescein isothiocyanate (FITC)/propidium iodide (PI) Apoptosis Kit (Beyotime, Shanghai, China) was used to stain the cell suspension. Cell apoptosis rate was detected using a flow cytometer (BD Biosciences, San Jose, CA, USA).

\section{Western blot (WB) analysis}

Total proteins were extracted using RIPA Lysis and Extraction Buffer (Thermo Scientific, Waltham, MA, USA). Extracted proteins $(20 \mu \mathrm{g})$ were separated on $10 \%$ SDS-PAGE gel and transferred onto PVDF membranes (Millipore, Billerica, MA, USA). Subsequent to blocking in $5 \%$ skim milk for $1 \mathrm{~h}$, the membranes were incubated with primary antibodies targeting B-cell lymphoma-2 (Bcl-2; 1:1,000, Boster, Wuhan, China), Bcl-2 associated x (Bax; 1:1,000, Boster), Cleaved-caspase3 (C-caspase3; 1:1,000, Boster), E2F3 (1:2,000, Boster) or GAPDH (1:4,000, Boster) overnight at $4^{\circ} \mathrm{C}$, and then interacted with HRP-conjugated secondary antibodies $(1: 10,000$, Boster) for $2 \mathrm{~h}$ at room temperature. Finally, protein signals were visualized using Enhanced Chemiluminescent Kit (Boster).

\section{Biotin-labeled RNA pull-down assay}

Biotin-labeled circHIPK3 probe and oligonucleotide (oligo) probe were obtained by Invitrogen. The probes were incubated with streptavidin magnetic beads (Thermo Scientific) 
to generate probe-coated beads. SRA01/04 cells were lysed and cell lysates were collected. Then, the probe-coated beads were incubated with cell lysates at $4^{\circ} \mathrm{C}$ overnight, followed by eluted and performed qRT-PCR. The primers of candidate miRNA were synthesized by Invitrogen.

\section{Dual-luciferase reporter assay}

The predicted sequences and mutant sequences of miR-499a-5p on circHIPK3 or E2F3 3'UTR were sub-cloned into the psiCHECK-2 reporter vector (Promega, Madison, WI, USA) to generate the wild-type (WT) and mutant-type (MUT) circHIPK3 or E2F3 3'UTR reporter vectors (circHIPK3 WT/MUT or E2F3 3'UTR WT/MUT), respectively. Lipofectamine 3000 was used to transfect with the reporter vectors and miR-NC or miR-499a-5p into SRA01/04 cells. After $48 \mathrm{~h}$, the luciferase activities were analyzed by Dual-Luciferase Assay Kit (Promega).

\section{Statistical analysis}

All experiments were repeated 3 times, and each independent experiment was set 3 times to take the average value. All data were presented as mean \pm standard deviation. Student's $t$-test and one-way analysis of variance were used for comparisons, and Pearson correlation analysis was used for analyzing the correlation. Statistical analyses were executed using GraphPad Prism 8.0 (GraphPad Software, La Jolla, CA, USA). $p$ value of less than 0.05 was considered to be statistically significant.

\section{Results}

CircHIPK3 was downregulated in the anterior lens capsule tissues of ARC patients By analyzing the genomic locus of circHIPK3 using the circPrimer software 
(http://www.bioinf.com.cn/), it was found that circHIPK3 was derived from the Exon 2 of HIPK3 gene, and its splicing maturity sequence length was 1099 bp (Fig. 1A). In the anterior lens capsule tissues of ARC patients, circHIPK3 expression was significantly lower than in normal anterior lens capsule tissues $(p=0.003$, Fig. 1B). To determine the authenticity of the circHIPK3, ActD assay and RNsae R assay were performed. As shown in Fig. 1C-D, compared to linear HIPK3, circHIPK3 was more stable and could resist the digestion of RNase $\mathrm{R}(p<0.001)$. Using the subcellular fractionation assay, we detected the expression of circHIPK3 in the nuclear and cytoplasm and confirmed that circHIPK3 was mainly distributed in the cytoplasm of SRA01/04 cells $(p<0.001$, Fig. 1E).

CircHIPK3 silencing inhibited the viability and promoted the apoptosis of SRA01/04 cells

To confirm the function of circHIPK3, we transfected with si-circHIPK3 into SRA01/04 cells. QRT-PCR results showed that si-circHIPK3 could markedly suppress the expression of circHIPK3 in SRA01/04 cells, confirming the transfection was successful ( $p<0.001$, Fig. 2A). CCK8 assay results indicated that circHIPK3 silencing obviously repressed the viability of SRA01/04 cells ( $p<0.001$, Fig. 2B). Besides, the results of flow cytometry assay suggested that the apoptosis rate of SRA01/04 cells could be promoted by circHIPK3 knockdown $(p<$ 0.001, Fig. 2C). Additionally, the protein levels of apoptosis markers were determined by WB analysis and the results presented that silenced circHIPK3 significantly reduced the Bcl-2 level and remarkably enhanced the Bax and C-caspase3 protein levels ( $p<0.001$, Fig. 2D). All data revealed that circHIPK3 knockdown could promote the progression of ARC.

\section{CircHIPK3 could sponge miR-499a-5p}


In order to find the targeted miRNAs of circHIPK3, we constructed a circHIPK3 probe and proved that it could significantly recruit circHIPK3 ( $p<0.001$, Fig. 3A). Subsequently, we used Starbase and Circbank tools to jointly predict the targeted miRNAs of circHIPK3, and identified 26 targeted miRNAs through Venn Diagram software analysis (Fig. 3B). Biotin-labeled RNA pull-down assay results showed that of the 26 predicted miRNAs, 22 miRNAs could be pulled down by the circHIPK3 probe, and we selected the miR-499a-5p with the highest expression for our study ( $p<0.001$, Fig. 3C). The Starbase tool predicted the targeting binding sites between circHIPK3 and miR-499a-5p were shown in Fig. 3D. Then, dual-luciferase reporter assay indicated that miR-499a-5p mimic significantly decreased the luciferase activity of the circHIPK3 WT vector without affecting that of the circHIPK3 MUT vector ( $p<0.001$, Fig. 3E). Meanwhile, our data also indicated that circHIPK3 silencing could markedly enhance miR-499a-5p expression in SRA01/04 cells $(p<0.001$, Fig. 3F). The ActD assay results suggested that circHIPK3 could accelerate the degradation of miR-499a-5p ( $p<0.001$, Fig. 3G). In addition, we found that miR-499a-5p expression was markedly increased in the anterior lens capsule tissues of ARC patients $(p<0.001$, Fig. $3 \mathrm{H})$, and its expression was negatively correlated with circHIPK3 expression $(p<0.001$, Fig. 3I).

Inhibition of miR-499a-5p partially reversed the effect of circHIPK3 silencing on the viability and apoptosis of SRA01/04 cells

Then, we co-transfected with si-circHIPK3 and anti-miR-499a-5p into SRA01/04 cells to explore whether circHIPK3 regulated ARC progression through sponging miR-499a-5p. The detection results of miR-499a-5p expression revealed that anti-miR-499a-5p could reverse the promotion effect of si-circHIPK3 on miR-499a-5p expression, indicating that the transfection 
efficiency of both was good ( $p<0.001$, Fig. 4A). By measuring the viability of cells using CCK8 assay, we discovered that miR-499a-5p inhibitor could partially reverse the suppressive effect of circHIPK3 knockdown on the viability of SRA01/04 cells $(p<0.001$, Fig. 4B). Moreover, flow cytometry results showed that the increasing effect of circHIPK3 silencing on the apoptosis of SRA01/04 cells also could be inverted by miR-499a-5p inhibitor $(p<0.001$, Fig. 4C). The results of WB analysis revealed that miR-499a-5p inhibitor also reversed the inhibition effect of circHIPK3 knockdown on Bcl-2 protein level and the promoting effect on Bax and C-caspase 3 protein levels in SRA01/04 cells $(p<0.001$, Fig. 4D). Therefore, all data suggested that circHIPK3 could sponge miR-499a-5p to regulate ARC progression.

\section{E2F3 was a target of miR-499a-5p}

For searching the targets of miR-499a-5p, we used the Starbase and Targetscan tools and found that E2F3 3'UTR had binding sites with miR-499a-5p (Fig. 5A). The results of dual-luciferase reporter assay showed that the luciferase activity of E2F3 3'UTR WT vector could be inhibited by miR-499a-5p overexpression compared to miR-NC, while the luciferase activity of E2F3 3'UTR MUT vector had not any changed ( $p<0.001$, Fig. 5B). Besides, we uncovered that miR-499a-5p overexpression could markedly repress the mRNA and protein levels of E2F3 in SRA01/04 cells ( $p<0.001$, Fig. 5C-D). Through detecting the expression of E2F3 expression in ARC tissues, we discovered that E2F3 expression was decreased in the anterior lens capsule tissues of ARC patients compared to normal anterior lens capsule tissues in the mRNA and protein levels ( $p<0.001$, Fig. 5E-F). Also, the results of Pearson correlation analysis revealed that there was a negative relationship between E2F3 expression and 
miR-499a-5p expression in the anterior lens capsule tissues of ARC patients $(p<0.001$, Fig. $5 G)$.

E2F3 overexpression partially reversed the effect of miR-499a-5p on the viability and apoptosis of SRA01/04 cells

To verify whether E2F3 participated in the regulation of miR-499a-5p on ARC progression, miR-499a-5p mimic and E2F3 overexpression vector were co-transfected into SRA01/04 cells. Compared with the miR-499a-5p + vector group, the increased E2F3 mRNA and protein expression levels in the miR-499a-5p + E2F3 group confirmed the effectiveness of miR-499a-5p mimic and E2F3 overexpression plasmid transfection ( $p<0.001$, Fig. 6A-B). By detecting the viability and apoptosis of SRA01/04 cells using CCK8 assay and flow cytometry, we found that miR-499a-5p overexpression could suppress the viability and increase the apoptosis of SRA01/04 cells, while this effect could be partially reversed by E2F3 overexpression ( $p<0.001$, Fig. 6C-D). In addition, miR-499a-5p also could decrease the protein level of Bcl-2 and improve the protein levels of Bax and C-caspase-3 in SRA01/04 cells. However, the effects of miR-499a-5p mimic on the protein levels of apoptosis markers also could be reversed by E2F3 overexpression ( $p<0.001$, Fig. 6E). Hence, these results confirmed that miR-499a-5p regulated ARC progression by targeting E2F3.

\section{CircHIPK3 regulated E2F3 by sponging miR-499a-5p}

Correlation analysis indicated that E2F3 expression was positively correlated with circHIPK3 expression in the anterior lens capsule tissues of ARC patients ( $p<0.001$, Fig. 7A). To further confirm the regulation of circHIPK3 on E2F3, we detected E2F3 expression in SRA01/04 cells transfected with si-circHIPK3 and anti-miR-499a-5p. As presented in Fig. 7B-C, we 
uncovered that circHIPK3 knockdown could suppress the mRNA and protein levels of E2F3, while this effect could be reversed by miR-499a-5p inhibitor $(p<0.001)$. Our data suggested that circHIPK3 sponged miR-499a-5p to positively regulate E2F3 expression.

\section{Discussion}

Lens turbidity and cloudiness are considered to be the characteristic manifestations of ARC [1, 2]. Although it is well known that the abnormal apoptosis of LECs is closely related to the occurrence of ARC, the specific mechanism is still unclear. Increasing evidence suggests that circRNA is a key regulator of cell biological processes, including cell viability and apoptosis $[11,12]$. However, there are few related studies on circRNA in ARC. In our study, we discovered that circHIPK3 was lowly expressed in ARC tissues, which was consistent with the previous study [17]. In addition, we found that circHIPK3 knockdown could restrain the viability and enhance the apoptosis of SRA01/04 cells. Hence, we speculated that the low expression of circHIPK3 in ARC might contribute to the development of ARC.

There are many mechanisms of circRNA, among which the most studied is act as competitive endogenous RNA of miRNA $[18,19]$. After the prediction of bioinformatics software and preliminary experiments, we determined that miR-499a-5p could be sponged by circHIPK3. MiR-499a-5p has been reported as a differentially expressed miRNA in a variety of diseases and has been shown to be associated with cell proliferation, differentiation and apoptosis [20-22]. Zong et al. reported that miR-499a-5p overexpression could inhibit the proliferation and promote the apoptosis of glioma [23], and Yang et al. showed that circ_0079593 knockdown could promote miR-499a-5p expression to suppress glioma proliferation and enhance apoptosis [24]. Therefore, miR-499a-5p may be an important 
miRNA that regulates cell biology process. In this study, we found that miR-499a-5p was highly expressed in ARC, and its inhibitor could reverse the suppressive effect of circHIPK3 knockdown on the viability and the promotion effect on the apoptosis of SRA01/04 cells. In addition, we also uncovered that miR-499a-5p overexpression could repress the viability and accelerate the apoptosis of SRA01/04 cells. Therefore, we speculated that miR-499a-5p might play a positive role in the progression of ARC, mainly by promoting LECs apoptosis.

$\mathrm{E} 2 \mathrm{~F} 3$ is a member of the transcription factor E2F family and is involved in the regulation of cell cycle processes [25]. A large number of studies have confirmed that E2F3 is highly expressed in many cancers, mainly promoting cell proliferation and inhibiting cell apoptosis [26]. The results of Xiang et al. indicated that E2F3 silencing could result in the apoptosis of SRA01/04 cells [27], and Gong et al. showed that E2F3 was underexpressed in ARC and its overexpression could enhance the viability and hinder the apoptosis of SRA01/04 cells [28]. Consistent with the previous studies, we discovered that E2F3 expression was decreased in ARC. Further experiments confirmed that miR-499a-5p contributed to the viability inhibition and apoptosis of SRA01/04 cells by inhibiting E2F3 expression. In addition, we also determined that circHIPK3 could regulate E2F3 by sponging miR-499a-5p. Our data suggested that circHIPK3 and E2F3 had a decrease expression, while miR-499a-5p had an elevated expression in the in the anterior lens capsule tissues of ARC patients compared with that in control. Also, correlation analysis suggested that circHIPK3 expression was positively correlated with E2F3 expression, while miR-499a-3p expression was negatively correlated with circHIPK3 or E2F3 expression. These data further revealed that circHIPK3 sponged miR-499a-5p to positively regulate E2F3. This was a new mechanism to reveal how 
circHIPK3 regulated ARC progression.

Similarly, in a recent study, circHIPK3 was shown to promote the proliferation and inhibit the apoptosis of LECs, thereby protecting LECs from dysfunction [29], which was consistent with our findings. However, Cui et al. proposed that circHIPK3 regulated the proliferation and apoptosis of LECs mainly through the regulation of the miR-221-3p/PI3K/AKT axis [29], indicating that circHIPK3 could mediate the proliferation and apoptosis of LECs by regulating the multiple pathways. Therefore, further research is necessary.

In general, our study showed that silencing of circHIPK3 inhibited the viability and promoted the apoptosis of LECs by mediating the miR-499a-5p/E2F3 axis, which provided new insights into the pathogenesis of ARC. In addition, our study pointed out that circHIPK3 might be a new target for the treatment of ARC, which will help promote the development of targeted therapy for ARC in clinical.

\section{Acknowledgment}

None

\section{Disclosure of interest}

The authors declare that they have no conflicts of interest.

\section{Funding}

None

\section{Reference}

1. Asbell PA, Dualan I, Mindel J, Brocks D, Ahmad M, Epstein S. Age-related cataract. Lancet. 2005;365:599-609. 
2. Fukuoka H, Afshari NA. The impact of age-related cataract on measures of frailty in an aging global population. Curr Opin Ophthalmol. 2017;28:93-97.

3. Sella R, Afshari NA. Nutritional effect on age-related cataract formation and progression. Curr Opin Ophthalmol. 2019;30:63-69.

4. Khosa T, Aslam S, Mustafa S, Akbar A, Shaikh RS, Iqbal F. Association of Single Nucleotide Polymorphisms in XRCC1 (194) and XPD (751) with Age-related cataract. Int Ophthalmol. 2018;38:1135-1146.

5. Shiels A, Hejtmancik JF. Mutations and mechanisms in congenital and age-related cataracts. Exp Eye Res. 2017;156:95-102.

6. Su D, Hu S, Guan L, Wu X, Shi C, Yang X, et al. Down-regulation of GJA3 is associated with lens epithelial cell apoptosis and age-related cataract. Biochem Biophys Res Commun. 2017;484:159-164.

7. Song E, Sun H, Xu Y, Ma Y, Zhu H, Pan CW. Age-related cataract, cataract surgery and subsequent mortality: a systematic review and meta-analysis. PLoS One. 2014;9:e112054.

8. Tseng VL, Greenberg PB, Wu WC, Jiang L, Li E, Kang JM, et al. Cataract surgery complications in nonagenarians. Ophthalmology. 2011;118:1229-1235.

9. Chen LL, Yang L. Regulation of circRNA biogenesis. RNA Biol. 2015;12:381-388.

10. Hsiao KY, Sun HS, Tsai SJ. Circular RNA - New member of noncoding RNA with novel functions. Exp Biol Med (Maywood). 2017;242:1136-1141.

11. Zhang HD, Jiang LH, Sun DW, Hou JC, Ji ZL. CircRNA: a novel type of biomarker for cancer. Breast Cancer. 2018;25:1-7. 
12. Salzman J. Circular RNA Expression: Its Potential Regulation and Function. Trends Genet. 2016;32:309-316.

13. Guo N, Liu XF, Pant OP, Zhou DD, Hao JL, Lu CW. Circular RNAs: Novel Promising Biomarkers in Ocular Diseases. Int J Med Sci. 2019;16:513-518.

14. Shan K, Liu C, Liu BH, Chen X, Dong R, Liu X, et al. Circular Noncoding RNA HIPK3 Mediates Retinal Vascular Dysfunction in Diabetes Mellitus. Circulation. 2017;136:1629-1642.

15. Lu H, Han X, Ren J, Ren K, Li Z, Sun Z. Circular RNA HIPK3 induces cell proliferation and inhibits apoptosis in non-small cell lung cancer through sponging miR-149. Cancer Biol Ther. 2020;21:113-121.

16. Zhang Y, Cao L, Jia J, Ye L, Wang Y, Zhou B, et al. CircHIPK3 is decreased in preeclampsia and affects migration, invasion, proliferation, and tube formation of human trophoblast cells. Placenta. 2019;85:1-8.

17. Liu X, Liu B, Zhou M, Fan F, Yu M, Gao C, et al. Circular RNA HIPK3 regulates human lens epithelial cells proliferation and apoptosis by targeting the miR-193a/CRYAA axis. Biochem Biophys Res Commun. 2018;503:2277-2285.

18. Li R, Jiang J, Shi H, Qian H, Zhang X, Xu W. CircRNA: a rising star in gastric cancer. Cell Mol Life Sci. 2020;77:1661-1680.

19. Shabaninejad Z, Vafadar A, Movahedpour A, Ghasemi Y, Namdar A, Fathizadeh H, et al. Circular RNAs in cancer: new insights into functions and implications in ovarian cancer. J Ovarian Res. 2019;12:84.

20. Neshati V, Mollazadeh S, Fazly Bazzaz BS, de Vries AAF, Mojarrad M, Naderi-Meshkin 
H, et al. MicroRNA-499a-5p Promotes Differentiation of Human Bone Marrow-Derived Mesenchymal Stem Cells to Cardiomyocytes. Appl Biochem Biotechnol. 2018;186:245-255.

21. Sun JC, Zheng B, Sun RX, Meng YK, Wang SM, Yang HS, et al. MiR-499a-5p suppresses apoptosis of human nucleus pulposus cells and degradation of their extracellular matrix by targeting SOX4. Biomed Pharmacother. 2019;113:108652.

22. He S, Li Z, Yu Y, Zeng Q, Cheng Y, Ji W, et al. Exosomal miR-499a-5p promotes cell proliferation, migration and EMT via mTOR signaling pathway in lung adenocarcinoma. Exp Cell Res. 2019;379:203-213.

23. Zong Z, Song Y, Xue Y, Ruan X, Liu X, Yang C, et al. Knockdown of LncRNA SCAMP1 suppressed malignant biological behaviours of glioma cells via modulating miR-499a-5p/LMX1A/NLRC5 pathway. J Cell Mol Med. 2019;23:5048-5062.

24. Yang Z, Li C, Fan XY, Liu LJ. Circular RNA circ_0079593 promotes glioma development through regulating KPNA2 expression by sponging miR-499a-5p. Eur Rev Med Pharmacol Sci. 2020;24:1288-1301.

25. DeGregori J, Johnson DG. Distinct and Overlapping Roles for E2F Family Members in Transcription, Proliferation and Apoptosis. Curr Mol Med. 2006;6:739-748.

26. Zhao W, Wang S, Qin T, Wang W. Circular RNA (circ-0075804) promotes the proliferation of retinoblastoma via combining heterogeneous nuclear ribonucleoprotein $\mathrm{K}$ (HNRNPK) to improve the stability of E2F transcription factor 3 E2F3. J Cell Biochem. 2020;121:3516-3525.

27. Xiang W, Lin H, Wang Q, Chen W, Liu Z, Chen H, et al. miR34a suppresses 
proliferation and induces apoptosis of human lens epithelial cells by targeting E2F3. Mol Med Rep. 2016;14:5049-5056.

28. Gong W, Li J, Wang Y, Meng J, Zheng G. miR-221 promotes lens epithelial cells apoptosis through interacting with SIRT1 and E2F3. Chem Biol Interact. 2019;306:39-46.

29. Cui G, Wang L, Huang W. Circular RNA HIPK3 regulates human lens epithelial cell dysfunction by targeting the miR-221-3p/PI3K/AKT pathway in age-related cataract. Exp Eye Res. 2020;198:108128. 


\section{Figure legends}

Fig. 1 The expression and identification of circHIPK3 in ARC. (A) The information of circHIPK3 was shown. (B) The expression of circHIPK3 in ARC tissues (ARC) and normal anterior lens capsule tissues (Control) was detected by qRT-PCR. (C) ActD assay was used to determine the stability of circHIPK3 and linear HIPK3 in SRA01/04 cells. (D) RNase R assay was employed to confirm the circular characteristic of circHIPK3 in SRA01/04 cells. (E) The expression levels of circHIPK3, U6 and GAPDH in the nuclear and cytoplasm of SRA01/04 cells were measured by qRT-PCR.

Fig. 2 Effects of circHIPK3 silencing on the viability and apoptosis of SRA01/04 cells. SRA01/04 cells were transfected with si-NC or si-circHIPK3. (A) The expression of circHIPK3 was detected by qRT-PCR to evaluate transfection efficiency. (B) CCK8 assay was used to measure the viability of cells. (C) The apoptosis of cells was determined using flow cytometry. (D) WB analysis was used to assess the protein levels of Bcl-2, Bax and C-caspase-3.

Fig. 3 CircHIPK3 could sponge miR-499a-5p. (A) The expression of circHIPK3 was measured by qRT-PCR to confirm the effectiveness of the circHIPK3 probe. (B) The Venn Diagram software analyzed the number of targeted miRNAs identified by Starbase and Circbank tools. (C) Biotin-labeled RNA pull-down assay was used to confirm the candidate miRNAs that could be pulled down by the circHIPK3 probe. (D) According to the prediction of Starbase tool, the binding sites and mutate sites between circHIPK 3 and miR-499a-5p were shown. (E) Dual-luciferase reporter assay was used to verify the interaction between circHIPK3 and miR-499a-5p. (F) The expression of miR-499a-5p was detected by qRT-PCR 
in SRA01/04 cells transfected with si-NC or si-circHIPK3. (G) ActD assay was employed to explore the effect of circHIPK3 on the degradation of miR-499a-5p. (H) QRT-PCR was used to determine the expression of miR-499a-5p in ARC tissues (ARC) and normal anterior lens capsule tissues (Control). (I) The correlation between circHIPK3 and miR-499a-5p was analyzed using Pearson correlation analysis.

Fig. 4 Effects of circHIPK3 silencing and miR-499a-5p inhibitor on the viability and apoptosis of SRA01/04 cells. SRA01/04 cells were transfected with si-NC, si-circHIPK3, si-circHIPK3 + anti-miR-NC or si-circHIPK3 + anti-miR-499a-5p. (A) QRT-PCR was used to determine the expression of miR-499a-5p to evaluate transfection efficiency. (B) The viability of cells was detected by CCK 8 assay. (C) The apoptosis of cells was measured by flow cytometry. (D) The protein levels of Bcl-2, Bax and C-caspase-3 were detected by WB analysis.

Fig. 5 E2F3 was a target of miR-499a-5p. (A) According to the prediction of Starbase tool, the binding sites and mutate sites between miR-499a-5p on E2F3 3'UTR were presented. (B) The interaction between E2F3 and miR-499a-5p was confirmed using dual-luciferase reporter assay. The mRNA and protein levels of E2F3 were measured by qRT-PCR (C) and WB analysis (D) in SRA01/04 cells transfected with miR-NC or miR-499a-5p mimic. QRT-PCR (E) and WB analysis (F) were used to detect the mRNA and protein levels of E2F3 in ARC tissues (ARC) and normal anterior lens capsule tissues (Control). (G) The correlation between E2F3 and miR-499a-5p was assessed by Pearson correlation analysis.

Fig. 6 Effects of miR-499a-5p and E2F3 overexpression on the viability and apoptosis of SRA01/04 cells. SRA01/04 cells were transfected with miR-NC, miR-499a-5p, miR-499a-5p 
+ vector or miR-499a-5p + E2F3. The mRNA and protein levels of E2F3 were determined using qRT-PCR (A) and WB analysis (B) to assess transfection efficiency. (C) The viability of cells was measured by CCK 8 assay. (D) The apoptosis of cells was determined by flow cytometry. (E) WB analysis was employed to measure the protein levels of Bcl-2, Bax and C-caspase-3.

Fig. 7 CircHIPK3 regulated E2F3 by sponging miR-499a-5p. (A) Pearson correlation analysis was performed to evaluate the correlation between E2F3 and circHIPK3. SRA01/04 cells were transfected with si-NC, si-circHIPK3, si-circHIPK3 + anti-miR-NC or si-circHIPK3 + anti-miR-499a-5p. QRT-PCR (B) and WB analysis (C) were used to measure the mRNA and protein levels of E2F3. 
A UCSC Genome Browser on Human Feb. 2009 (GRCh37/hg19) Assembly



D

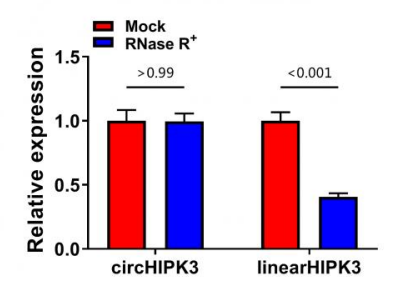

E

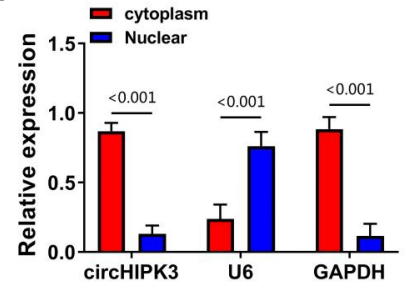

B



C





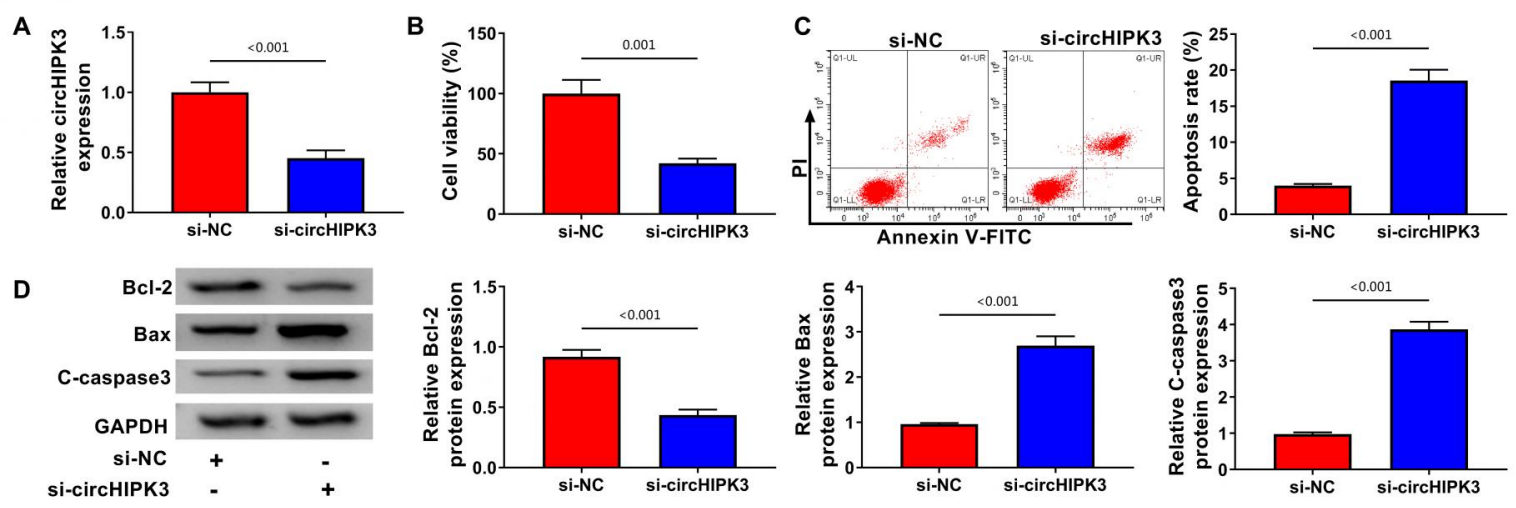
A

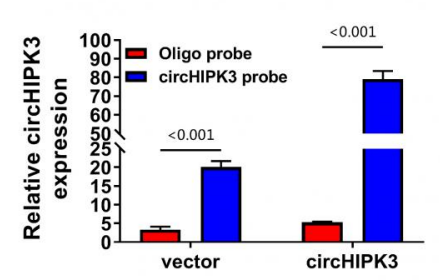

B

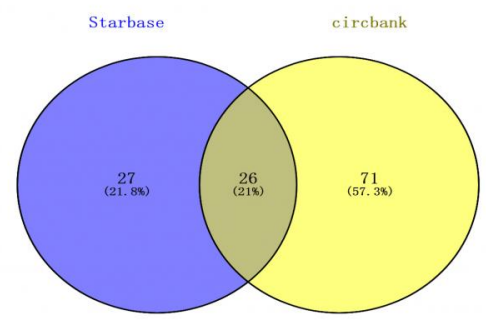

C

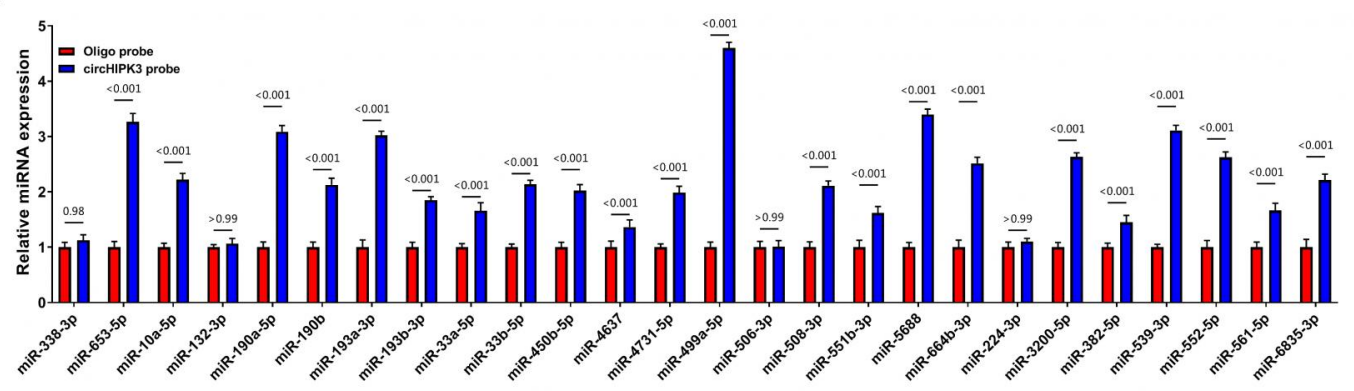

D

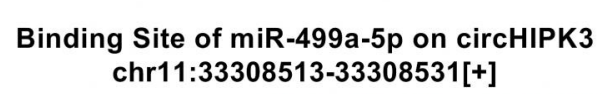
circHIPK3 WT 5 ' guACAGCA--UG-.-AAGUCUUAu

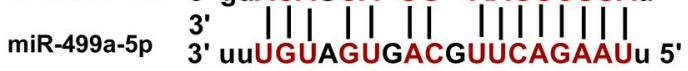
circHIPK3 MUT 5' guGACGAG.-CA---CGACUGCGu 3'

G

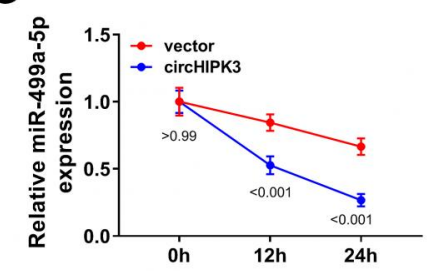

E

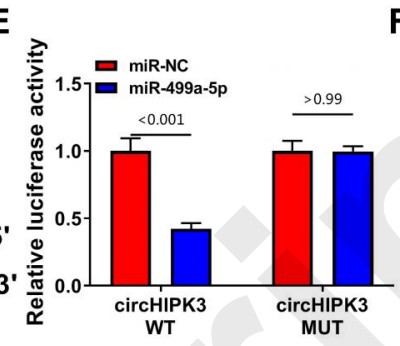

F

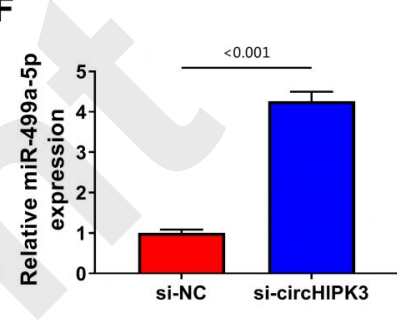

H



I

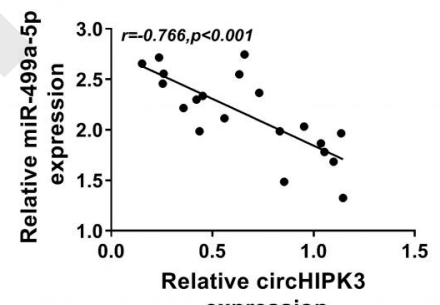

expression 


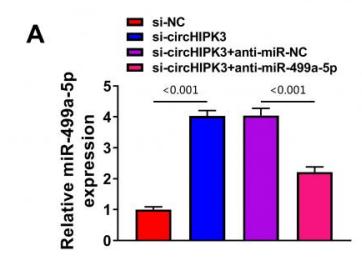

D

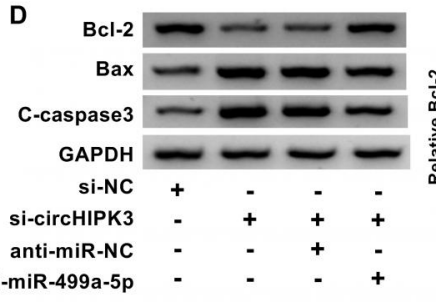

anti-miR-499a-5p

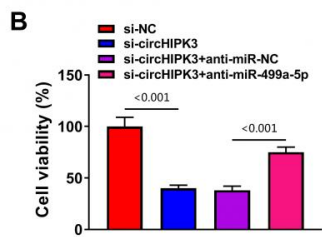

믈 ${ }_{\text {si-circhlPH }}^{\text {si- }}$

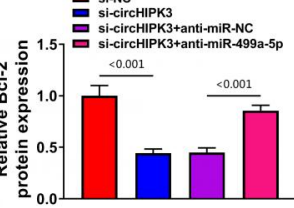

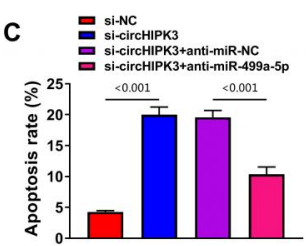

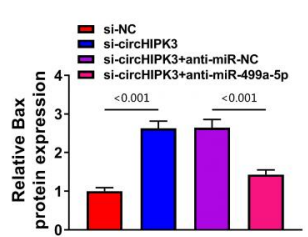

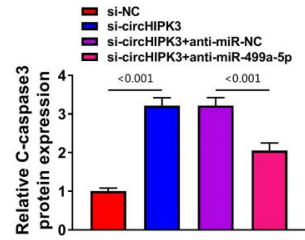


A

Binding Site of miR-499a-5p on E2F3 chr6:20492947-20492954[+]



B

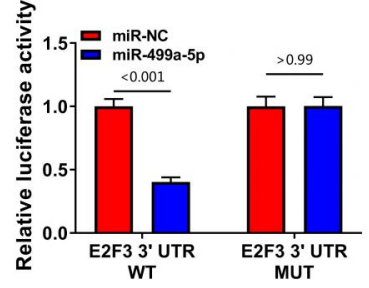

C

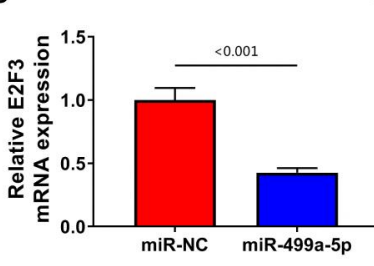

E

$$
\text { E2F3 }
$$

GAPDH
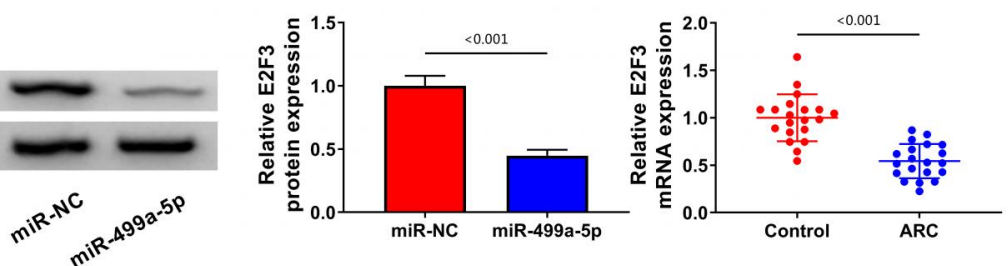

$\mathbf{F}$

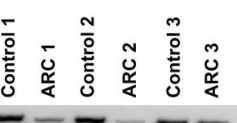

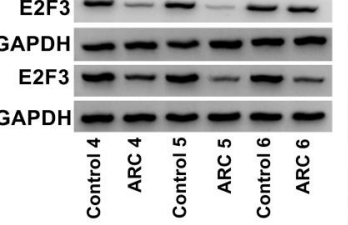

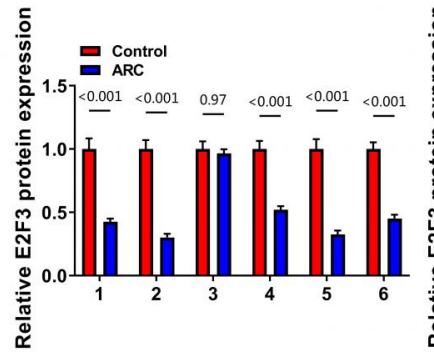

$\mathbf{G}$

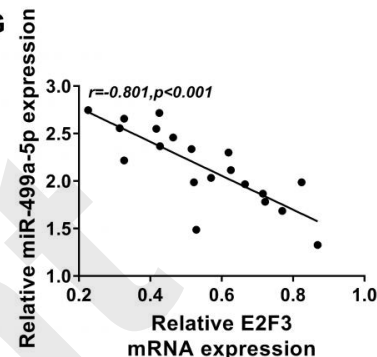



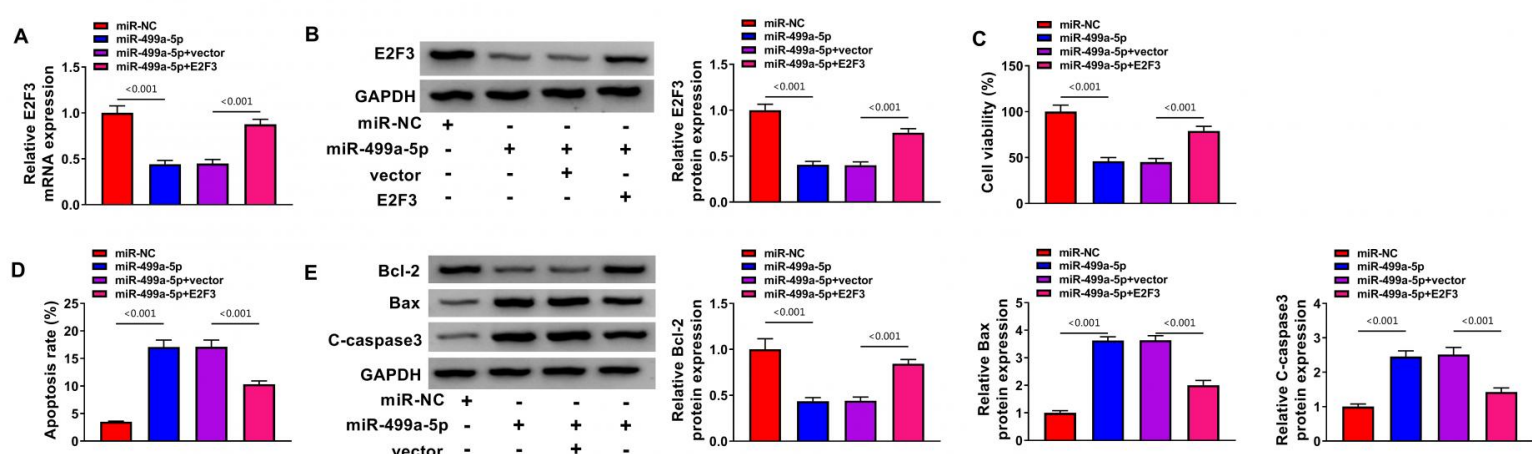

R-499a-5p
vector

vector : 




C

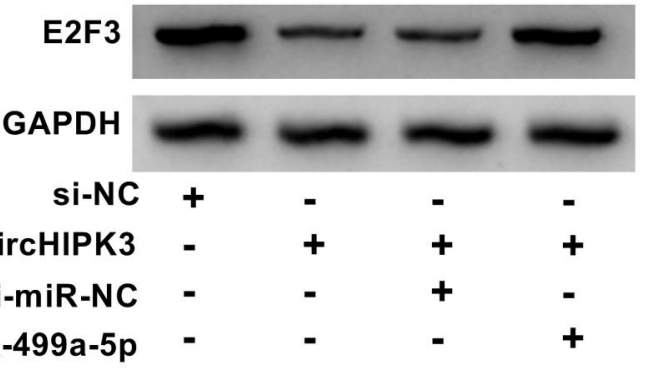

B
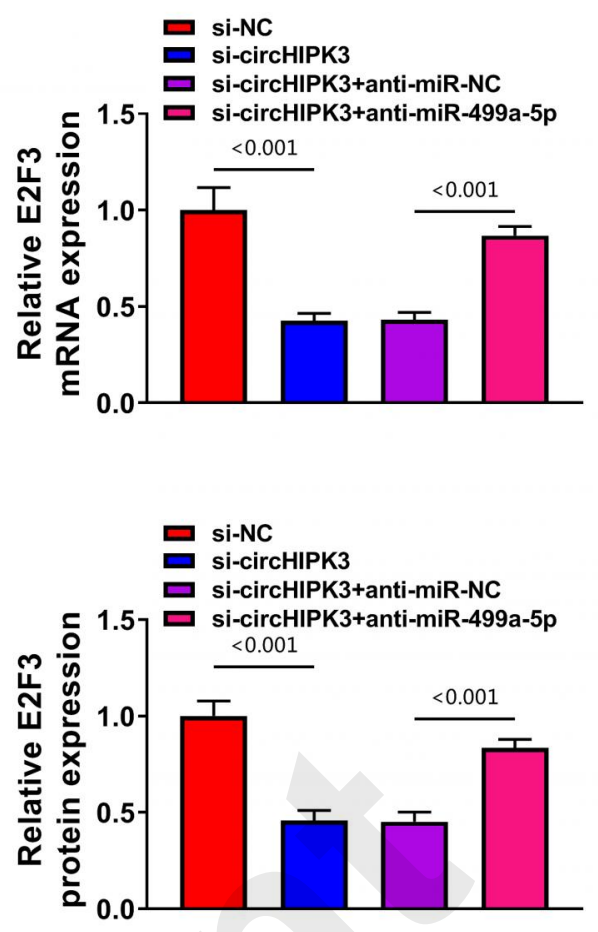